\title{
A mutation in the $L A M C 2$ gene causes the Herlitz junctional epidermolysis bullosa (H-JEB) in two French draft horse breeds
}

\author{
Dragan Milenkovic, Stéphane Chaffaux, \\ Sead TAOURIT, Gérard GuÉrin*
}

Laboratoire de génétique biochimique et de cytogénétique,

Département de génétique animale, Institut national de la recherche agronomique, Centre de recherches de Jouy, 78352 Jouy-en-Josas Cedex, France

(Received 2 August 2002; accepted 5 September 2002)

\begin{abstract}
Epidermolysis bullosa (EB) is a heterogeneous group of inherited diseases characterised by skin blistering and fragility. In humans, one of the most severe forms of EB known as Herlitz-junctional EB (H-JEB), is caused by mutations in the laminin 5 genes. EB has been described in several species, like cattle, sheep, dogs, cats and horses where the mutation, a cytosine insertion in exon 10 of the $L A M C 2$ gene, was very recently identified in Belgian horses as the mutation responsible for JEB. In this study, the same mutation was found to be totally associated with the JEB phenotype in two French draft horse breeds, Trait Breton and Trait Comtois. This result provides breeders a molecular test to better manage their breeding strategies by genetic counselling.
\end{abstract}

horse / LAMC2 / epidermolysis bullosa / laminin 5

\section{INTRODUCTION}

Epidermolysis bullosa (EB) is a heterogeneous group of mechano-bullous disorders characterised by fragility of the skin and the mucous membranes. In humans, EB is divided into three different forms depending on the level of the separation within the dermo-epidermal junction as recognised by diagnostic transmission electron microscopy $[8,9]$. The junctional form of EB, JEB, is characterised by blister formation within the lamina lucida of the basement membrane zone and by an autosomal recessive pattern of inheritance. In the severe Herlitz variant, H-JEB, tissue cleavage results from the mutations in one

* Correspondence and reprints

E-mail: guerin@jouy.inra.fr 
of the three genes (LAMA3, LAMB3 or LAMC2) $[1,15,20]$ encoding the three subunits $(\alpha 3, \beta 3$ and $\gamma 2)$ of the extracellular adhesion ligand laminin 5 associated with the hemidesmosome-anchoring filament complexes. Likewise, cases of EB have been described in different species, such as sheep [4], dogs [19], cats [18], mice [5], and rats [2].

In the horse, a case of possible hereditary epitheliogenesis imperfecta was first described by two clinicians in 1935 [3]. Since then, several publications referring to epidermolysis bullosa or closely related clinical diagnoses have been reported. These cases of tegument defects appear in different breeds such as the Belgian draft horses [14] but also in a crossing between a Thoroughbred and a Quarter Horse [6] or in Saddlebred horses [7]. The cases in Belgian foals were defined as junctional EB with limited hereditary evidence [10,13]. All cases in draft horses involved skin malformation resulting in blisters probably caused by an abnormal epidermal-dermal junction. Different areas of the body were affected, in particular the limbs with recurrent loss of the hooves. The affected foals died or were euthanised. In France, a group of lethal junctional EB was described for the first time in the Trait Breton and Trait Comtois draft horses and the limited family data are compatible with a recessive mode of inheritance [11]. In a very recent study, a cytosine insertion in exon 10 of the $L A M C 2$ gene [21], was identified as being responsible for JEB in Belgian horses. This study was aimed at checking if the same mutation is the cause of the disease in two French breeds, the Trait Breton and Trait Comtois draft horses.

\section{METHODS}

Clinical cases of JEB were collected through the Haras Nationaux, equine veterinarians and breed associations. The animals were of Trait Breton and Trait Comtois draft horse breeds suffering predominantly from multifocal absence of the skin. Due to the lethal prognosis, the foals were euthanised in general a few days after birth. The histological analyses of the skin were performed in a single anatomo-pathology laboratory (Laboratoire d'anatomie pathologique vétérinaire, Amboise, France). The skin was fixed in formalin and then embedded in paraffin. The sections were cut and either stained with eosin and hematoxylin or with periodic acid-Schiff (PAS) for the basement membrane zone observation.

Pedigrees were obtained from the breeders providing the animals and the SIRENET database (http://www.harasire.net). Blood was collected from 66 horses and genomic DNA was purified from the peripheral blood according to standard protocols. The panel included horses from two breeds: 19 Trait Comtois ( 5 affected foals, 8 mares, 3 sires and 3 control horses presumably non-carriers unrelated to the families with the JEB cases), and 47 Trait Breton (10 affected foals, 15 mares, 10 sires and 12 control horses). 
The presence of the mutation, a cytosine insertion in exon 10 of the $L A M C 2$ gene, described by [21], was revealed by PCR on genomic DNA of our panel using primers 5'-TGTTACTCAGGGGATGAGAA-3' and 5'-CTGGGGGCAGTTATTGCAC-3' followed by polyacrylamide gel electrophoresis. PCR was performed as advised by the authors with one of the primers labelled with $\gamma$ - $\left[{ }^{33} \mathrm{P}\right]$-dATP by T4 polynucleotide kinase (Promega) in a Perkin-Elmer Cetus 9600 or MJ Research PTC100 thermocycler. For sequencing, PCR amplification of a $170 \mathrm{bp}$ fragment was performed in the same PCR conditions and $5 \mu \mathrm{L}$ of the products were electrophoresed on $2 \%$ agarose gels. The fragments were purified with a QIAquick PCR purification kit (Qiagen) and were sequenced on an ABI 377 automated sequencer (Applied Biosystems).

Linkage disequilibrium between the JEB phenotype and the mutation was analysed using the maximum likelihood method of [22]. Two-point analyses were performed using the DISLAMB programme [22] obtained from the Rockefeller Institute website (http://linkage.rockefeller.edu).

Total RNA was isolated from skin biopsies obtained from one control and from one heterozygous individual, using the TRIZOL Reagent (Invitrogen). First strand cDNA was obtained from $2.5 \mu \mathrm{g}$ of total RNA using Superscript II RT (Invitrogen) as recommended by the manufacturer. Two microliters of the reaction mixture were used in PCR amplification of exon 10 of LAMC2 cDNA using the primers identified by [21], one of the primers labelled with $\gamma-\left[{ }^{33} \mathrm{P}\right]-\mathrm{dATP}$ by T4 polynucleotide kinase (Promega) in a Perkin-Elmer Cetus 9600 or MJ Research PTC100 thermocycler, followed by polyacrylamide gel electrophoresis.

\section{RESULTS}

The phenotype of the affected foals suggests a condition in horses similar to H-JEB in humans. Affected foals were born with skin blistering, skin and buccal ulceration followed by the loss of the hooves, as ascertained by a veterinarian and confirmed by histological examination (Fig. 1). The affected skin showed disjunction of the epidermis from the underlying dermis at the dermal-epidermal junction. PAS tests were performed demonstrating that lamina densa adheres to the dermis (Fig. 2).

The insertion of a cytosine at position 1368-1369 of the cDNA of LAMC2 (AY082802) [21], was tested in all horses of the panel. In the draft horses, electrophoresis on a polyacrylamide gel revealed two PCR fragments for mares and sires, one for affected foals and a distinctively smaller one in all controls (Fig. 3). The presence of the mutation was ascertained by sequencing of the PCR fragment in all horses from the panel. Similarly, all presumably healthy non-carrier horses were homozygous for the wild type, while all affected foals 

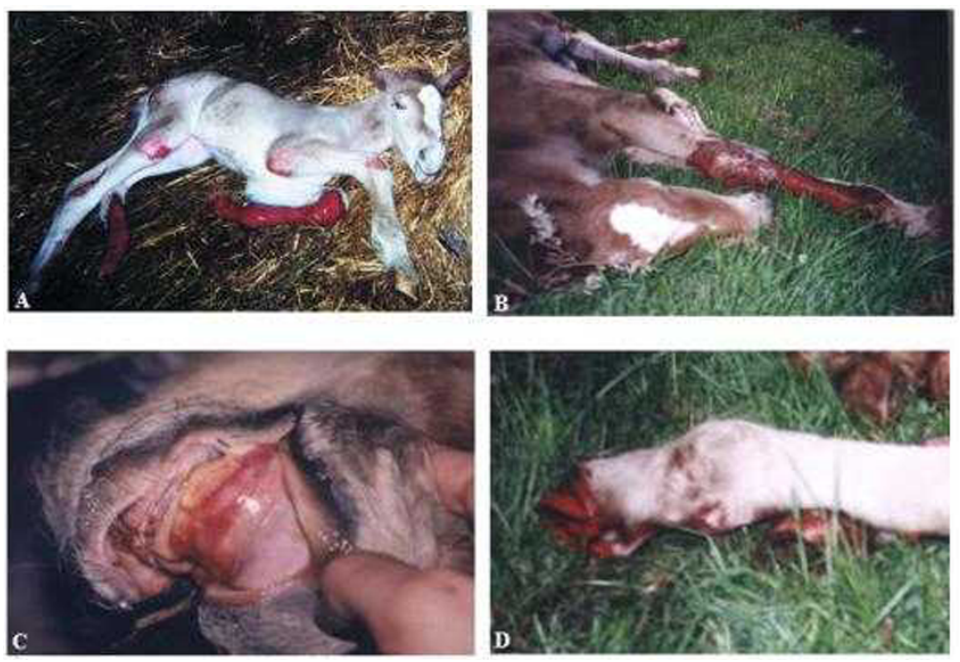

Figure 1. Clinical features of horse epidermolysis bullosa in affected foals. (A and B) Extensive absence of skin is visible, principally on the lower limbs and joints; (C) buccal cavity involvement (ulcer of gum mucosa) is also observed and (D) loss of the hoof.

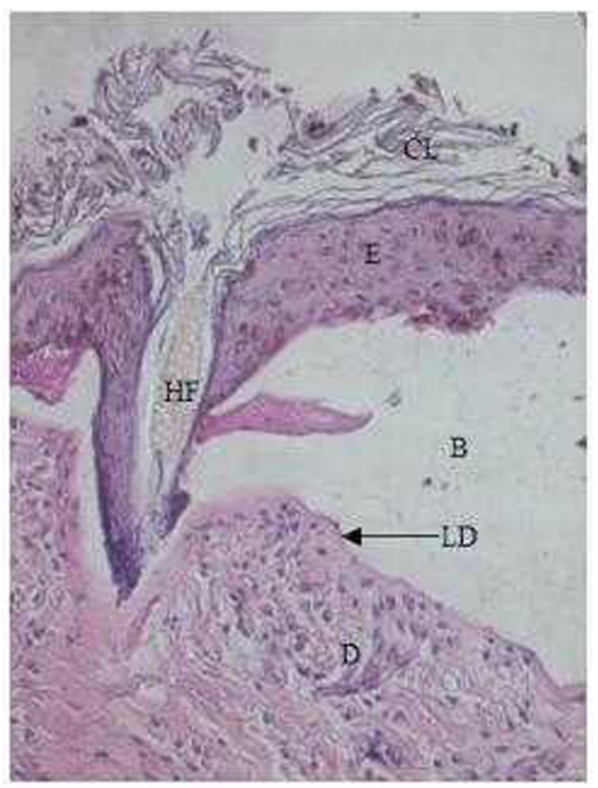

Figure 2. Transverse histological skin section of horse junctional epidermolysis bullosa skin. The separation between the dermis (D) and epidermis (E) giving rise to a blister (B) is observed. It is a lesion caused by a defective basement membrane zone. The lamina densa (LD) is visible. Cornified layer cells (CL) and a hair follicle (HF) are also observed (H\&E; X40). 


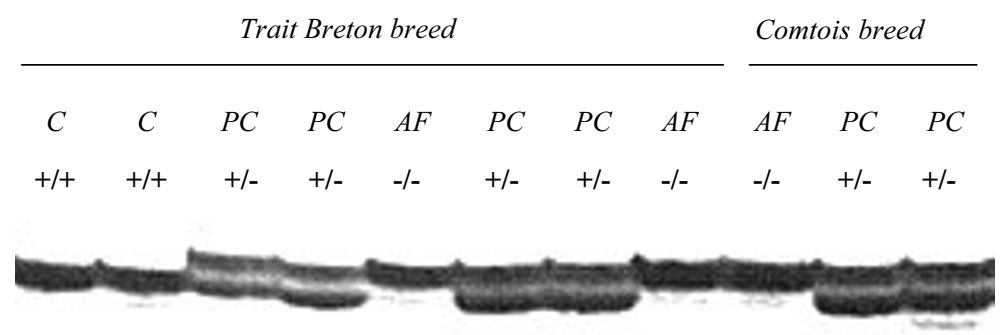

Figure 3. Genotyping of one nucleotide insertion mutation on polyacrylamide gel of radioactive PCR products spanning a $170 \mathrm{bp}$ region of exon 10 of the $L A M C 2$ gene. A small fragment is observed in the controls (C), a larger one in the affected foals (AF), and both for healthy parental carriers (PC).

were homozygous for the insertion and all parents were heterozygous. The 1368-1369insC mutation showed a total linkage disequilibrium with the JEB in the two French draft breeds. The $P$-value of the LRT test was $6.8 \times 10^{-14}$, with a chi-square value of 54.85 (lambda $=1)$, and a $P$-value of $1.1 \times 10^{-11}$, for a chi-square test value of 46.14 .

Semi quantification of the LAMC2 mRNA was performed by RT-PCR on mRNA obtained from the skin of one control and one heterozygous horse. Electrophoresis on a polyacrylamide gel of amplified exon 10 of LAMC2 cDNA revealed one band in the control corresponding to the wild form and two bands in a heterozygous horse. The band corresponding to the wild form was stronger than that of the mutated form (data not shown).

\section{DISCUSSION}

The clinical description of the disease in the French draft affected foals collected in this study was similar to those observed in other species. In humans, widespread blistering of the skin, stratified mucous membranes and the epidermis detachment from the dermis at the basal membrane level characterise the lethal form of JEB observed just after birth. In the Trait Breton and Trait Comtois French draft horse breeds, JEB is characterised by a multifocal absence of the epidermis predominantly involving the lower limbs with multiple ulceration of the skin. The absence of the mucosal epithelium and ulceration of the buccal cavity was also observed. Separation of the coronary bands from the hoof wall resulted in the loss of the hooves (Fig. 1). This clinical feature of JEB was confirmed by a histological analysis showing the epidermal detachment from the dermis at the basal membrane level (Fig. 2). These observations are consistent with the reports on H-JEB in humans and JEB in Belgian horses. In addition, our data were in accordance with the recessive mode of inheritance observed in humans and horses. 
The presence of a single mutation can be explained by the pedigree structure of our panel and the history of the three draft breeds. Pedigrees of the propositus were reconstituted as far back as possible, sometimes up to the seventh generation. In each of the Comtois and Breton breeds, one or two stallions, probably heterozygous for the mutation, were found in the pedigree of all the affected foals, revealing the existence of common sires in the families of the same breed, as well as common ancestors for the two breeds. Similarly, it is known that the Comtois and the Belgian breeds share common ancestors. This explains how a single mutation that probably occurred in a founder animal could have spread within and among the populations of different breeds.

Two lines of observations provide evidence that the 1368-1369insC mutation is responsible for the disorder in the Trait Breton and Trait Comtois. A complete association was found between the mutation and the phenotype with a significant linkage disequilibrium $(P<0.01)$. Furthermore, the insertion of a cytosine provokes a frameshift in the open reading frame and generates a premature termination codon. The truncated $\gamma 2$ subunit lacks its C-terminal domain that mediates the interaction with the two other subunits, $\alpha 3$ and $\beta 3$, of laminin 5 preventing its formation as well as that of anchoring filament complexes.

The low level of the mutant allele cDNA of a heterozygous animal supports the possible explanation of a rapid mRNA decay of the mutant allele. This agrees with the lack of detection of the laminin $\gamma 2$ protein by a specific antibody in the tongue epithelia of an affected foal [21]. The possible rapid decay of the mutated $\gamma 2$ mRNA could be explained by the degradation of mRNA containing a premature termination codon via the "nonsense-mediated mRNA decay pathway" $[12,16,17]$.

The identification of the causal mutation of JEB in the Trait Breton and Trait Comtois is of great importance to draft horse breeders. A rapid, simple genotyping method by DNA amplification from blood or hair samples detecting normal and mutated fragments is now available for genetic counselling. The identification of healthy carriers for the mutation allows to develop different breeding strategies. Populations can be conducted, avoiding matings between carriers to obtain unaffected foals. Alternatively, breeder associations may decide to eradicate the mutation by preventing all carriers to reproduce.

In conclusion, this study (i) demonstrates that the LAMC2 mutation detected in the Belgian breed is also responsible for JEB in the Trait Breton and Trait Comtois; (ii) confirms by clinical and histological analysis that EB in these two breeds is homologous to human H-JEB; (iii) proposes that genetic counselling based on a rapid molecular test for the identification of healthy carriers will help breeders to circumvent the disorder in their population; and (iv) favours the hypothesis of a rapid mRNA decay of the LAMC2 mutated allele. Still, a number of questions need to be addressed: the existence of different forms 
of epidermolysis in the horse, their incidence in different breeds and the identification of the causal mutations.

\section{ACKNOWLEDGEMENTS}

We would like to thank the Anatomo-pathology Laboratory (Dr. Vet. Jean Loï LeNet) for histological examination and for providing the slides of skin, as well as numerous veterinarians, breeders, breeder associations and the Haras nationaux for helping us gather the information of JEB cases in these horses. The primer sequences were kindly provided by Dr. G. Meneguzzi (Inserm U385, Faculté de Médecine, Nice, France) and the photographs of the diseased animals by Dr. Vet. Watrin and Dr. Vet. Lagrue. This study was partially supported by the Haras nationaux and D. Milenkovic was supported by a fellowship from Inra.

\section{REFERENCES}

[1] Aumailley M., Smyth N., The role of laminins in basement membrane function, J. Anat. 193 (1998) 1-21.

[2] Brenneman K.A., Olivry T., Dorman D.C., Rudimentary hemidesmosome formation in congenital generalized junctional epidermolysis bullosa in the Sprague-Dawley rat, Vet. Pathol. 37 (2000) 4336-4339.

[3] Brethelsen H., Eriksson K., Epitheliogenesis imperfecta neonatorum in a foal, possibly of a hereditary nature, J. Comp. Path. Ther. 48 (1935) 258-297.

[4] Bruckner-Tuderman L., Guscetti F., Ehrensperger F., Animal model for dermolytic mechanobullous disease: sheep with recessive dystrophic epidermolysis bullosa lack collagen VII, J. Invest. Dermatol. 96 (1991) 452-458.

[5] Colognato H., Yurchenco P.D., The laminin alpha2 expressed by dystrophic $\operatorname{dy}(2 \mathrm{~J})$ mice is defective in its ability to form polymers, Curr. Biol. 22 (1999) $1327-1330$.

[6] Crowell W.A., Stephenson C., Gogger H.S., Epitheliogenesis imperfecta in a foal, J. Am. Vet. Med. Assoc. 168 (1976) 56-58.

[7] Dubielzig R.R., Wilson J.W., Beck K.A., Robbins T., Dental dysplasia and epitheliogenesis imperfecta in a foal, Vet. Pathol. 23 (1986) 325-327.

[8] Fine J.D., Bauer E.A., Briggaman R.A., Carter D.M., Eady R.A., Esterly N.B., Holbrook K.A., Hurwitz S., Johnson L., Lin A., et al., Revised clinical and laboratory criteria for subtypes of inherited epidermolysis bullosa. A consensus report by the Subcommittee on Diagnosis and Classification of the National Epidermolysis Bullosa Registry, J. Am. Acad. Dermatol. 24 (1991) 119-135.

[9] Fine J.D., Eady R.A., Bauer E.A., Briggaman R.A., Bruckner-Tuderman L., Christiano A., Heagerty A., Hintner H., Jonkman M.F., McGrath J., McGuire J., Moshell A., Shimizu H., Tadini G., Uitto J., Revised classification system for inherited epidermolysis bullosa: Report of the Second International Consensus Meeting on Diagnosis and Classification of Epidermolysis Bullosa, J. Am. Acad. Dermatol. 42 (2000) 1051-1066. 
[10] Frame S.R., Harrington D.D., Fessler J., Frame P.F., Hereditary junctional mechanobullous disease in a foal, J. Am. Vet. Med. Assoc. 193 (1988) 1420-1424.

[11] Goureau J.M., Feillou C., Morand M., Courreau J.F., Dupere A.M., Alliot A., Épidermolyse bulleuse jonctionelle léthale chez le cheval de trait en France, Bull. Acad. Vét. Fr. 62 (1989) 345-353.

[12] Hood J., Exon-exon junction - what's your function?, Trends Cell Biol. 11 (2001) 463.

[13] Johnson G.C., Kohn C.W., Johnson C.W., Garry F., Scott D., Martin S., Ultrastructure of junctional epidermolysis bullosa in Belgian foals, J. Comp. Pathol. 99 (1998) 329-336.

[14] Kohn C.W., Johnson G.C., Garry F., Johnson C.W., Martin S., Scott D.W., Mechanobullous disease in two Belgian foals, Equine Vet. J. 21 (1989) 297-301.

[15] Korge B.P., Krieg T., The molecular basis for inherited bullous diseases, J. Mol. Med. 74 (1996) 59-70.

[16] Lykke-Andersen J., Shu M.D., Steitz J.A., Communication of the position of exon-exon junctions to the mRNA surveillance machinery by the protein RNPS1, Science 293 (2001) 1836-1839.

[17] Nagy E., Maquat L.E., A rule for termination-codon position within introncontaining genes: when nonsense affects RNA abundance, Trends Biochem. Sci. 23 (1998) 198-199.

[18] Olivry T., Dunston S.M., Marinkovich M.P., Reduced anchoring fibril formation and collagen VII immunoreactivity in feline dystrophic epidermolysis bullosa, Vet. Pathol. 36 (1999) 616-618.

[19] Palazzi X., Marchal T., Chabanne L., Spadafora A., Magnol J.P., Memeguzzi G., Inherited dystrophic epidermolysis bullosa in inbred dogs: A spontaneous animal model for somatic gene therapy, J. Invest. Dermatol. 115 (2000) 135-137.

[20] Pulkkinen L., Uitto J., Mutation analysis and molecular genetics of epidermolysis bullosa, Matrix Biol. 18 (1999) 29-42.

[21] Spirito F., Charlesworth A., Linder K., Ortonne J.P., Baird J., Meneguzzi G., Animal models for skin blistering conditions: absence of laminin 5 causes hereditary junctional mechanobullous disease in the Belgian horse, J. Invest. Dermatol. 3 (2002) 684-691.

[22] Terwilliger J.D., A powerful likelihood method for the analysis of linkage disequilibrium between trait loci and one or more polymorphic marker loci, Am. J. Hum. Genet. 56 (1995) 777-787. 